\title{
Applying NPWT to bleeding open wounds after forefoot amputation in diabetic foot patients - a case report
}

\author{
Jacek Białecki, Przemysław Pyda, Anna Kołodziejska, Anna Rybak, Sebastian Sowier
}

\section{CASE REPORT}

\begin{abstract}
NPWT is increasingly used in patients with diabetic foot. The protocol for performing NPWT is subject to debate. Main concerns regard the type of suction to use (continuous, intermittent, or variable), the wound-packing material, or the exact pressure to apply. Typically, an optimum pressure range of $-\mathbf{8 0}$ to $-\mathbf{1 2 5} \mathrm{mmHg}$ is indicated. Following bone resection in diabetic foot patients, the surgical wound is left open, which often entails bleeding from the resection site. In these cases, the start of NPWT was typically delayed by $24-48$ hours - during that time a pressure dressing was applied - and NPWT was only started once bleeding had ceased. In order to initiate NPWT as soon as possible, we decided to start it at a higher negative pressure than usual, i.e. $-180 \mathrm{mmHg}$, expecting that this would stop the bleeding. Only then would we reduce the negative pressure. This paper presents the course of NPWT with high negative pressure values after an amputation in $\mathbf{2}$ diabetic foot patients. In both cases, our assumptions were confirmed. The patients did not bleed, the drained volume did not exceed 30 $\mathbf{m l}$ (which seems clinically insignificant) in the first $\mathbf{4 0}$ minutes of treatment. Later, with negative pressure at $-120 \mathrm{mmHg}$, no bleeding into the dressing was observed. Following the treatment (which lasted for 9 days), the wounds granulated normally, with no signs of inflammation. Applying VAC dressing using high negative pressure values to bleeding wounds immediately after surgery may stop the bleeding, enabling immediate initiation of NPWT.
\end{abstract}

Keywords-negative pressure wound therapy, diabetic foot, amputation, bleeding

\section{INTRODUCTION}

$\mathbf{N}$ EGATIVE pressure wound therapy (NPWT) is increasingly used in patients with diabetic foot, both in treating wounds caused by ulceration, and in healing surgical wounds following bone resection. NPWT assists in wound healing in a number of ways: it drains exudate, contracts the wound edges, alters blood flow in the wound edges, stimulates angiogenesis, reduces tissue edema, stimulates the formation of granulation tissue, creates a moist environment, and stimulates the wound bed ${ }^{1+\sqrt[3]{3}}$ Multiple studies indicate that NPWT is superior to other wound dressing methods

Manuscript received 09.09.2018; revised 21.12.2018. This work did not receive any financial support. Authors declare no conflict of interest.

Author affiliations: Department of General, Minimally Invasive and Trauma Surgery, Franciszek Raszeja City Hospital in Poznań, ul. Mickiewicza 2, 60-834 Poznań, Poland, (JB, PP, AK, AR, SS)

*Correspondence to: Jacek Białecki: jacekt.bialecki@gmail.com in the treatment of diabetic foot. ${ }^{4 / 6}$ The protocol for performing NPWT is subject to debate. Indications for when NPWT should and should not be used have been established quite clearly $] 7[8$ However, indications regarding the type of suction to use (continuous, intermittent, or variable), 1] 9011 the wound-packing material,, 12$] 13$ or the exact pressure to apply $\sqrt{14}-17$ are less definitive. Typically, an optimum pressure range of -80 to $-125 \mathrm{mmHg}$ is indicated, though other recommendations can also be found: Timmers et al. (2005) suggest, for instance, that the best wound healing outcomes can be achieved at pressures ranging from -250 to -300 $\mathrm{mmHg} \cdot{ }^{18}$ Following bone resection in diabetic foot patients, the surgical wound is left open, which often entails bleeding from the resection site. In these cases, the start of NPWT was typically delayed by $24-48$ hours - during that time a pressure dressing was applied - and NPWT was only started once bleeding had ceased. This was due to the widespread belief that applying NPWT immediately after the surgery might increase bleeding. However, in order to initiate NPWT as soon as possible, we decided to start it at a higher negative pressure than usual, i.e. $-180 \mathrm{mmHg}$, expecting that this would stop the bleeding. Only after achieving hemostasis would we reduce the negative pressure. This paper presents the course of NPWT with high negative pressure values after an amputation in 2 diabetic foot patients.

\section{CASE REPORT}

\section{A. Surgical interventions}

Patient A (53 years old), suffering from type-2 diabetes, hypertension, and coronary artery disease, was referred to our hospital with necrosis of the fourth toe and extensive phlegmon of the right foot. Initial debridement of the wound was performed on an outpatient basis: an amputation of the fourth toe was performed and the drainage of the subcutaneous forefoot tissue was initiated (Fig. 11. Subsequently, the patient was admitted to our hospital for further diagnostics and therapy. A blood culture test was run and a targeted antibiotic therapy was initiated (Ciprofloxacin 2 x $400 \mathrm{mg}$ i.v.). After 4 days of conservative treatment, clinical symptoms of ischemia in the distal parts of the limb were found. Therefore a computed tomography angiography of lower limbs was 


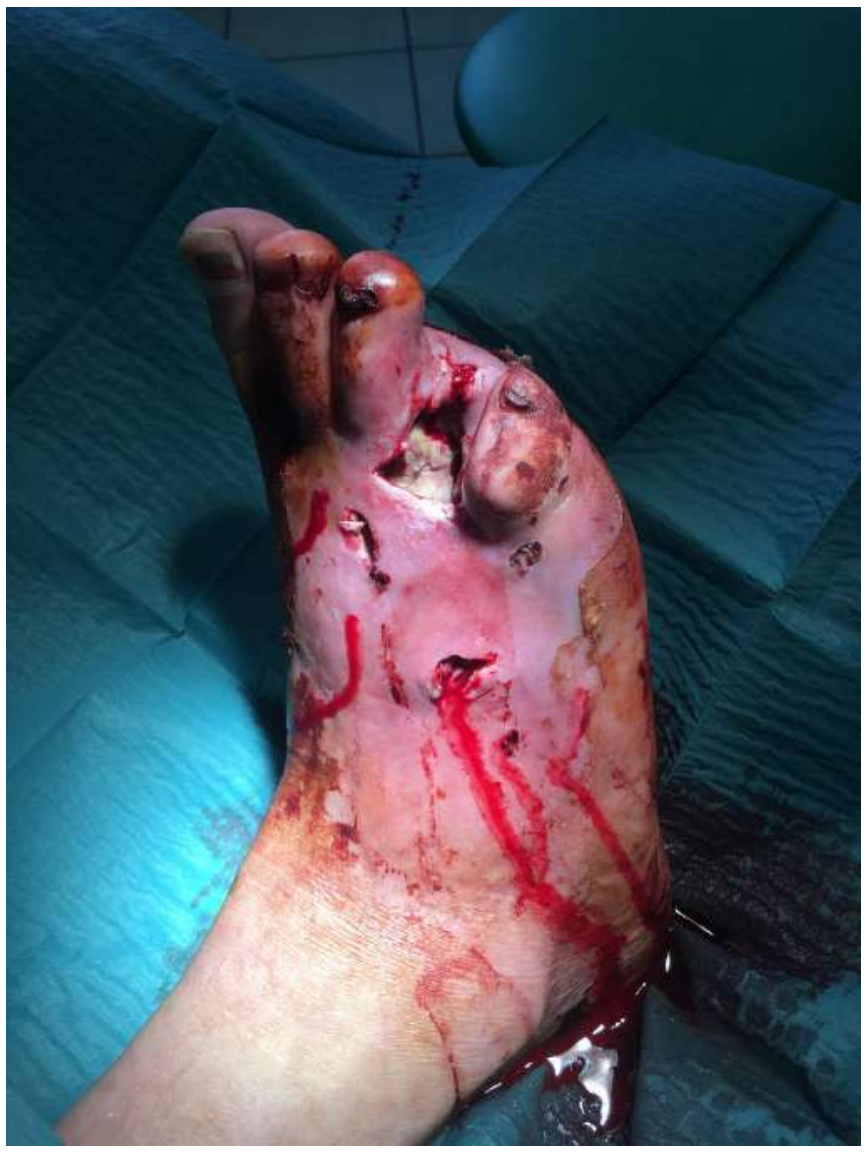

Figure 1. A wound after an amputation of the fourth toe. On the back of the foot, there are small incisions from drains placed in the outpatient clinic.

performed. A critical constriction of the superficial femoralartery of the left limb was discovered. A vascular reconstructive surgery was performed - reversed femoral-popliteal saphenous vein bypass. A proper blood supply was obtained, which was confirmed by a Doppler ultrasonography. In the first week post-operatively, an inflammatory progression occurred in the distal part of the limb. Extensive necrosis developed in the fifth toe and part of the subcutaneous tissue of the forefoot and sole. The patient underwent nonanatomical amputation of the fifth toe, and resection of the fourth and fifth metatarsophalangeal joints together with the distal parts of the fourth and fifth metatarsal bones (Fig. 2). A broad-spectrum antibiotic therapy was initiated (Tazocin 3 $\mathrm{x} 4.5 \mathrm{~g}$ i.v.). Throughout the entire hospital stay the patient continued insulin therapy (Insulatard: $16 \mathrm{u}-0 \mathrm{u}-28 \mathrm{u}$; NovoRapid: $16 \mathrm{u}-10 \mathrm{u}-8 \mathrm{u})$ and Clexane was administered subcutaneously (1 x $40 \mathrm{mg}$ ).

Patient B (55 years old), suffering from Charcot foot and diabetic foot ulcer, previously managed on an outpatient basis, was admitted to our hospital with extensive phlegmon in the left crus and foot. Crural amputation was performed, with standard suturing of the stump. Despite targeted antibiotic treatment (Imipenem $500 \mathrm{mg} /$ Cilastatin $500 \mathrm{mg}$ x 4 i.v.), 3 days post-operatively massive phlegmon developed in the stump tissues, with inflammatory infiltration of the soft tissue of the thigh. Surgical wound revision was performed, which

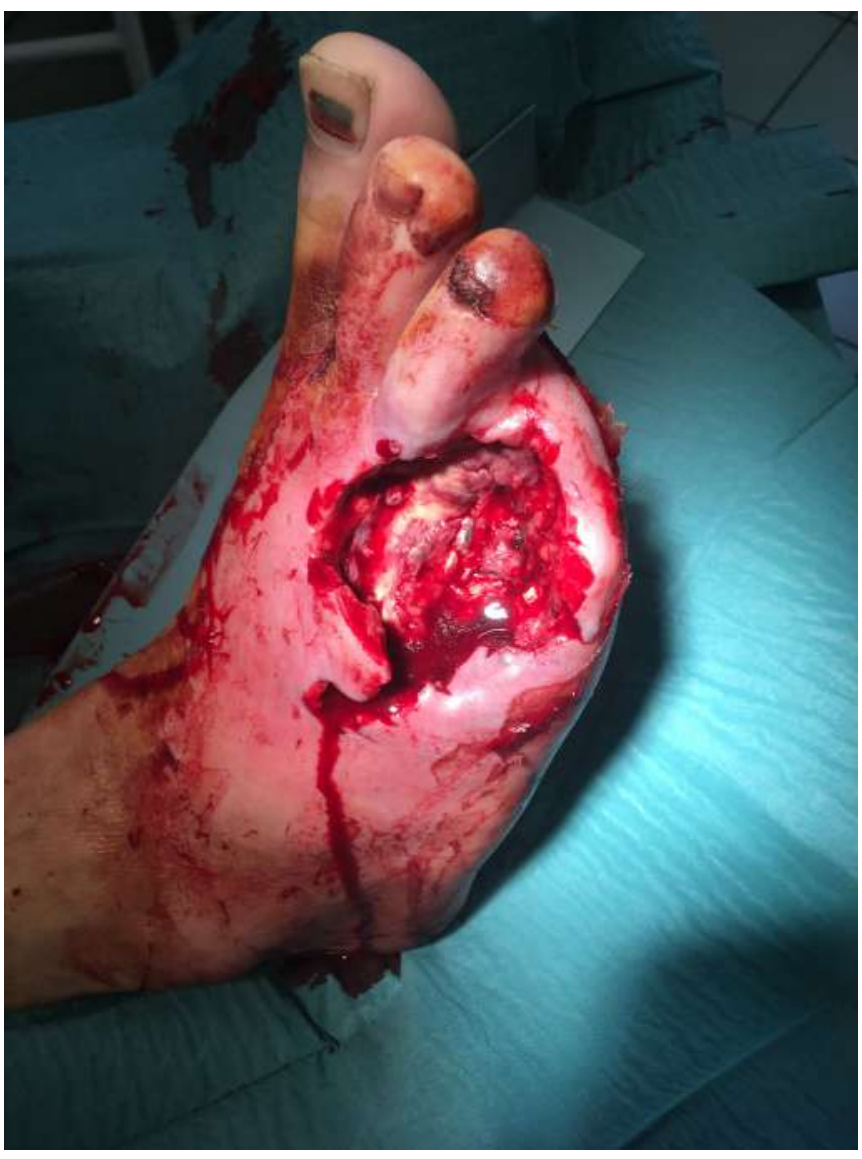

Figure 2. A bleeding wound after a non-anatomical amputation of the fifth toe, and resection of the fourth and fifth metatarsophalangeal joints together with the distal parts of the fourth and fifth metatarsal bones.

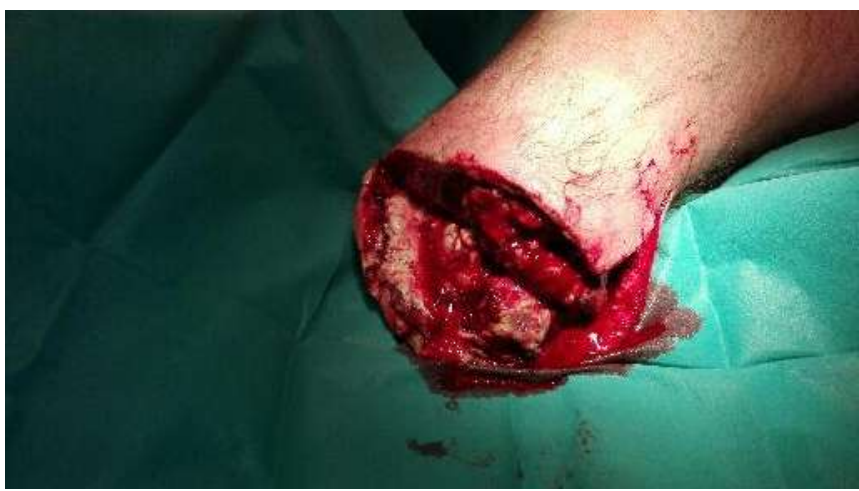

Figure 3. Revision of the crural stump following the amputation.

involved removing sutures from the skin and muscle, and dissecting the skin and subcutaneous tissue from the lateral part of the thigh along the inflammatory infiltrate. Pus was evacuated, and infected and necrotic tissue was removed (Fig. 3). Throughout the entire hospital stay the patient continued insulin therapy (Humulin N: $16 \mathrm{u}-0 \mathrm{u}-14 \mathrm{u}$; Humalog $14 \mathrm{u}$ $-12 \mathrm{u}-10 \mathrm{u}$ ) and Clexane was administered subcutaneously (1 x $100 \mathrm{mg})$. 


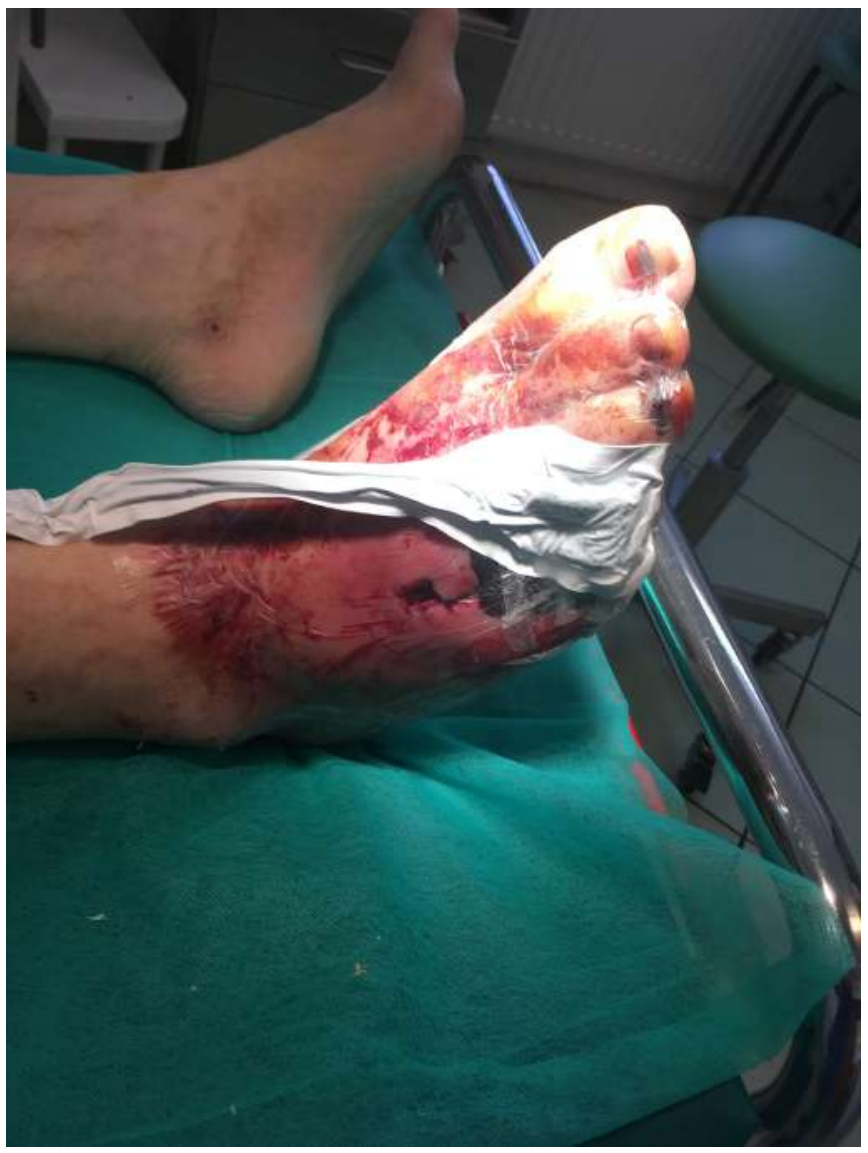

Figure 4. Foot after fourth and fifth metatarsal bone amputation, with the VAC dressing applied immediately after the procedure.

\section{B. NPWT treatment}

In both cases, despite parenchymatous bleeding from the surrounding tissue, a VAC dressing (Renasys-F, Ontario, Canada) was applied immediately after the surgery. A polyurethane foam dressing was cut to fit the wound (Fig. 4). The dressing was sealed and NPWT was started. A pressure of $-180 \mathrm{mmHg}$ was applied for the first 30 minutes after the procedure, then $-140 \mathrm{mmHg}$ for the next 10 minutes. Subsequently, the negative pressure was reduced to -120 $\mathrm{mmHg}$ and maintained until the end of treatment.

In both cases, our assumptions were confirmed. The patients did not bleed, the drained volume did not exceed $30 \mathrm{ml}$ (which seems clinically insignificant) in the first 40 minutes of treatment. Treatment continued for 3 days, after which the dressing was changed. Then, NPWT was continued for the next two 3-day cycles, with the dressing changed again on the 6th post-operative day. Throughout the entire 9-day treatment the wound did not bleed. The VAC dressing drained a mean volume of $40 \mathrm{ml}$ of serosanguineous exudate per day. Following the treatment, the wounds granulated normally, with no signs of inflammation (Fig. 5).

\section{DISCUSSION}

NPWT is now commonly used in the treatment of diabetic foot. Though general indications for the treatment have been

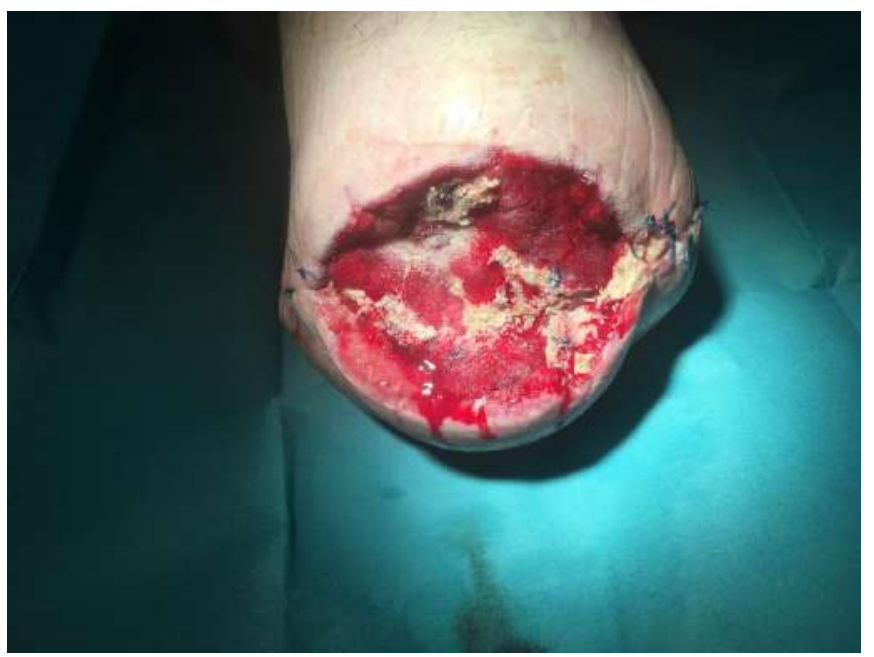

Figure 5. Wound site with VAC dressing removed after 9 days of NPWT.

established, no single protocol has been developed. Reports from a number of centers indicate that in cases of open wounds following amputation or bone resection in patients with diabetic foot, early start of NPWT may trigger or exacerbate bleeding. Hence, treatment start was typically delayed by 24-48 hours, until after hemostasis had been achieved. This is also the protocol recommended by some authors 19$] 20$ The standard method for the achievement of hemostasis is electrocoagulation. However, in cases of diabetic foot, this creates multiple new thermal necrosis sites, which is undesirable, particularly if infection exists. Another method for managing bleeding from the surgical wound involves applying a pressure dressing, which restricts blood flow to the tissues. However, this delays the start of NPWT, while the larger volume of secretions remaining in the wound increases treatment time. If the tissues surrounding the wound are infected, it is desirable to apply NPWT as soon as possible, which allows for early drainage of the secretions that inhibit wound healing due to the content of proinflammatory factors. To effectively remove the secretions from the wound, we decided to apply a VAC dressing onto the fresh wound, immediately after the surgery. Bearing in mind the concerns related to bleeding, we attempted to adjust the negative pressure in a way ensuring the evacuation of secretions without causing bleeding. Some studies on NPWT discuss the impact of the negative pressure in the dressing on blood supply to the surrounding tissue. (It should be noted that measurements to determine the optimum negative pressure value are performed on tissues with a specific compactness - therefore, their results should not be automatically used to prescribe pressure values for use in tissues with different properties. For instance, different pressures may be optimal for the abdominal wall and for the foot.) Wackenfors et al., studying the impact of negative pressure values ranging between -50 and $-200 \mathrm{mmHg}$ on microvascular blood flow around the wound in porcine models, reported that the use of NPWT may reduce microvascular blood flow in the tissues directly adjacent to the wound, and that the higher the negative pressure value, the greater the affected area 2122 
Based on this report and our own experience with NPWT, we decided to apply a negative pressure of $-180 \mathrm{mmHg}$ for the first 30 minutes, to prevent bleeding. The negative pressure was then reduced to $-140 \mathrm{mmHg}$ for the next 10 minutes. After that, treatment was continued at the standard pressure of $-120 \mathrm{mmHg}$. This allowed us to start NPWT immediately after the surgery without increasing the risk of bleeding. Further observations are required to develop a protocol for NPWT after amputations in diabetic foot patients.

\section{CONCLUSION}

Applying VAC dressing using high negative pressure values to bleeding wounds immediately after surgery may stop the bleeding, enabling immediate initiation of NPWT. Further observations are required to develop a protocol for NPWT after amputations in diabetic foot patients.

\section{REFERENCES}

[1] M. Malmsjö, L. Gustafsson, S. Lindstedt, B. Gesslein, and R. Ingemansson, "The effects of variable, intermittent, and continuous negative pressure wound therapy, using foam or gauze, on wound contraction, granulation tissue formation, and ingrowth into the wound filler," Eplasty, vol. 12, 2012.

[2] C. Huang, T. Leavitt, L. R. Bayer, and D. P. Orgill, "Effect of negative pressure wound therapy on wound healing," Current problems in surgery, vol. 51, no. 7, pp. 301-331, 2014.

[3] M. Y. Hasan, R. Teo, and A. Nather, "Negative-pressure wound therapy for management of diabetic foot wounds: a review of the mechanism of action, clinical applications, and recent developments," Diabetic foot \& ankle, vol. 6 , no. 1, p. 27618, 2015.

[4] S. Liu, C.-z. He, Y.-t. Cai, Q.-p. Xing, Y.-z. Guo, Z.-1. Chen, J.-1. $\mathrm{Su}$, and L.-p. Yang, "Evaluation of negative-pressure wound therapy for patients with diabetic foot ulcers: systematic review and metaanalysis," Therapeutics and clinical risk management, vol. 13, p. 533, 2017.

[5] A. M. Lone, M. I. Zaroo, B. A. Laway, N. A. Pala, S. A. Bashir, and A. Rasool, "Vacuum-assisted closure versus conventional dressings in the management of diabetic foot ulcers: a prospective case-control study," Diabetic foot \& ankle, vol. 5, no. 1, p. 23345, 2014.

[6] M. Sajid, Q. Mustafa, and N. Shaheen, "Comparison of negative pressure wound therapy using vacuum-assisted closure with advanced moist wound therapy in the treatment of diabetic foot ulcers." $J$ Coll Physicians Surg Pak, vol. 25, no. 11, pp. 789-93, 2015.

[7] B. Mrozikiewicz-Rakowska, E. Bucior, J. Kania, A. Nowak, M. Chojnowski, and J. Krzymień, "Modern alternative or first-line treatment: How to safely use negative pressure wound therapy in diabetic foot syndrome?" Negative Pressure Wound Therapy Journal, vol. 2, no. 1, 2015.

[8] H. Birke-Sorensen, M. Malmsjo, P. Rome, D. Hudson, E. Krug, L. Berg, A. Bruhin, C. Caravaggi, M. Chariker, M. Depoorter et al., "Evidence-based recommendations for negative pressure wound therapy: treatment variables (pressure levels, wound filler and contact layer)-steps towards an international consensus," Journal of Plastic, Reconstructive \& Aesthetic Surgery, vol. 64, pp. S1-S16, 2011.
[9] O. Borgquist, R. Ingemansson, and M. Malmsjö, "The effect of intermittent and variable negative pressure wound therapy on wound edge microvascular blood flow," Ostomy/wound management, vol. 56, no. 3, p. 60, 2010.

[10] C. Ahearn, "Intermittent npwt and lower negative pressures-exploring the disparity between science and current practice: a review." $O s$ tomy/wound management, vol. 55, no. 6, pp. 22-28, 2009.

[11] M. C. Lessing, R. B. James, and S. C. Ingram, "Comparison of the effects of different negative pressure wound therapy modes-continuous, noncontinuous, and with instillation-on porcine excisional wounds," Eplasty, vol. 13, 2013.

[12] O. Borgquist, L. Gustafsson, R. Ingemansson, and M. Malmsjö, "Micro-and macromechanical effects on the wound bed of negative pressure wound therapy using gauze and foam," Annals of plastic surgery, vol. 64, no. 6, pp. 789-793, 2010.

[13] M. Malmsjö, R. Ingemansson, R. Martin, and E. Huddleston, "Negative-pressure wound therapy using gauze or open-cell polyurethane foam: similar early effects on pressure transduction and tissue contraction in an experimental porcine wound model," Wound repair and regeneration, vol. 17, no. 2, pp. 200-205, 2009.

[14] O. Borgquist, R. Ingemansson, and M. Malmsjö, "Wound edge microvascular blood flow during negative-pressure wound therapy: examining the effects of pressures from-10 to-175 mmhg," Plastic and reconstructive surgery, vol. 125, no. 2, pp. 502-509, 2010.

[15] —, "The influence of low and high pressure levels during negativepressure wound therapy on wound contraction and fluid evacuation," Plastic and reconstructive surgery, vol. 127, no. 2, pp. 551-559, 2011.

[16] M. J. Morykwas, B. J. Faler, D. J. Pearce, and L. C. Argenta, "Effects of varying levels of subatmospheric pressure on the rate of granulation tissue formation in experimental wounds in swine," Annals of plastic surgery, vol. 47, no. 5, pp. 547-551, 2001

[17] O. Borgquist, E. Anesäter, E. Hedström, C. K. Lee, R. Ingemansson, and M. Malmsjö, "Measurements of wound edge microvascular blood flow during negative pressure wound therapy using thermodiffusion and transcutaneous and invasive laser d oppler velocimetry," Wound Repair and Regeneration, vol. 19, no. 6, pp. 727-733, 2011.

[18] M. S. Timmers, S. Le Cessie, P. Banwell, and G. N. Jukema, "The effects of varying degrees of pressure delivered by negative-pressure wound therapy on skin perfusion," Annals of plastic surgery, vol. 55 , no. 6, pp. 665-671, 2005.

[19] P. S. Nain, S. K. Uppal, R. Garg, K. Bajaj, and S. Garg, "Role of negative pressure wound therapy in healing of diabetic foot ulcers," Journal of surgical technique and case report, vol. 3, no. 1, 2011.

[20] M. Meloni, V. Izzo, E. Vainieri, L. Giurato, V. Ruotolo, and L. Uccioli, "Management of negative pressure wound therapy in the treatment of diabetic foot ulcers," World journal of orthopedics, vol. 6, no. 4, p. 387, 2015.

[21] A. Wackenfors, J. Sjögren, R. Gustafsson, L. Algotsson, R. Ingemansson, and M. Malmsjö, "Effects of vacuum-assisted closure therapy on inguinal wound edge microvascular blood flow," Wound repair and regeneration, vol. 12, no. 6, pp. 600-606, 2004.

[22] A. Wackenfors, R. Gustafsson, J. Sjögren, L. Algotsson, R. Ingemansson, and M. Malmsjö, "Blood flow responses in the peristernal thoracic wall during vacuum-assisted closure therapy," The Annals of thoracic surgery, vol. 79, no. 5, pp. 1724-1730, 2005. 